\title{
Turning process monitoring of internal combustion engine piston's cylindrical surface
}

\author{
Pawel Twardowski ${ }^{1}$, Maciej Tabaszewski ${ }^{2}$, and Szymon Wojciechowski ${ }^{1,{ }^{*}}$ \\ ${ }^{1}$ Poznan University of Technology, Faculty of Mechanical Engineering and Management, Institute of \\ Mechanical Technology, Poznan, Poland \\ 2 Poznan University of Technology, Faculty of Mechanical Engineering and Management, Institute of \\ Applied Mechanics, Poznan, Poland
}

\begin{abstract}
In this paper the monitoring method of turning process of combustion engine piston's cylindrical surface is proposed. During this process, the PCD diamond tool cuts the piston's cylindrical surface with the 3 grooves. The first groove is made in the cast iron insert. In case, when the machining allowances are selected inappropriately, the tool cuts the cast iron insert and consequently generates the reject. The proposed monitoring system based on vibration signals analysis enables the detection of these critical situations and react, in order to maintain the production quality.
\end{abstract}

\section{Introduction}

The chip material's removal during machining processes is accompanied by plastic-elastic deformations of work material induced by the ploughing mechanism [1,2], as well as the thermal phenomena in the cutting zone [3-5]. The appearance of these phenomena is strictly correlated with the generation of cutting forces and vibrations, which in turn can significantly affect the deterioration of the machined surface quality $[6,7]$. On the other hand, the force and vibration signals can be applied for the machining process monitoring.

The literature survey shows the examples of vibration signals' application to the monitoring of cutting tools' condition [8]. However, Scheffer et al. [9] proposed the machining process monitoring on the basis of the acoustic emission and cutting force signals. The application of this kind of systems is important, especially during the automated manufacturing, where the human's interference is limited. In above specified conditions, the problem related to the cutting tool's control becomes essential [10].

\section{Experimental details}

In this paper the monitoring method of turning process of combustion engine piston's cylindrical surface with the application of mechanical vibrations' measurement is proposed.

Figure 1 shows an image of the IC engine's piston with the visible grooves intended for the piston rings.

\footnotetext{
*Corresponding author: sjwojciechowski@o2.pl
} 

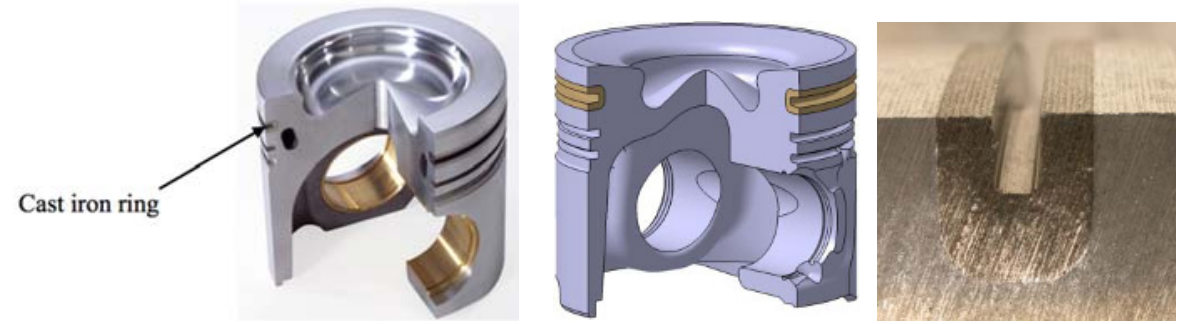

Fig. 1. View of piston internal combustion (IC) engine.

The first groove contains the cast iron insert, in order to strengthen its structure. This groove is fitted to the compression ring, which is responsible for the maintaining the appropriate pressure in the combustion chamber. The subsequent two rings are: scraper ring (the middle one) and oil control ring.

The investigated problem is connected with the positioning accuracy of cutting tool's working part in the special toolholder. In this instance, the cutting edge's positioning tolerances are contained in the narrow range equaled to $\pm 0.01 \mathrm{~mm}$. Exceeding these values during the machining process can lead to the collision between the PCD tool and cast iron insert. Furthermore, the additional factor which can contribute to the collision is the relatively low accuracy of the cast iron insert submerged inside the aluminum body (dimensional deviation higher than $0.01 \mathrm{~mm}$ ).

The first groove (together with the cast iron insert), intended for the compression ring is being machined before carrying out the preliminary turning of the piston's cylindrical surface. The longitudinal turning of the cylindrical surface is carried out with the application of PCD (polycrystalline diamond) tool. As it is well known, the PCD tool cannot be applied to the cutting of the ferritic materials, because of carbon diffusion into the iron alloys. Therefore, the machining allowances has been selected in order to avoid the tool's contact with the cast iron ring (which has been previously machined in the finishing conditions). Nevertheless, the instant contact of PCD tool with cast iron ring during machining is possible when the cutting depth is selected inappropriately, or the ring has been manufactured incorrectly. The piston manufacturing process is completely automated, thus the PCD tool's condition is not controlled. In case when the PCD tool will start to cut the cast iron ring, then the rapid growth of tool wear will occur, which can lead to the rupture of the intermetallic bonding between the aluminum alloy and cast iron ring. Consequently, the production shortcoming is being generated.

Therefore, the primary task of the proposed monitoring system is the detection of situation in which the PCD tool cuts the iron ring, and subsequently the immediate stoppage of the process, together with sending the appropriate message to the machine's control system. The primary subsystem of the developed monitoring system is the module intended to measurement and analysis of mechanical vibration signals (Figure 2).

The turning of the piston's cylindrical surface is carried out in the following steps:

- PCD tool is located outside the work piece,

- activation of rotational speed and feed motion,

- initiation of aluminum turning with the selected parameters (AB section-Figure 3),

- $\quad$ approach of tool to the cast iron ring (point B),

- $\quad$ if the machining allowance (depth of cut $a_{p}$ ) is appropriately selected, then tool is not contacting with the section BE (Figure 4),

- $\quad$ if the machining allowance is inappropriately selected, the tool cuts in the areas: $\mathrm{BC}$ and $\mathrm{DE}$ (tool cuts the cast iron - Figure 5),

- further machining of aluminum (section EF) until the tool's output (point F). 

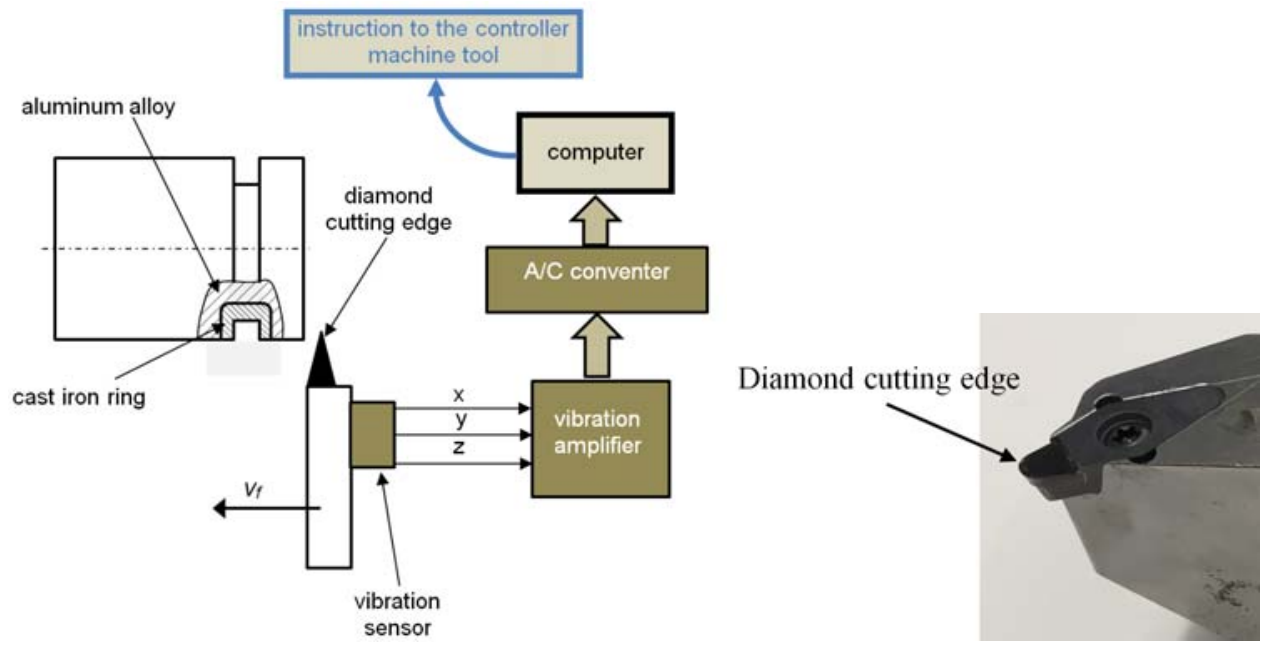

Fig. 2. The scheme of the proposed turning process monitoring system.
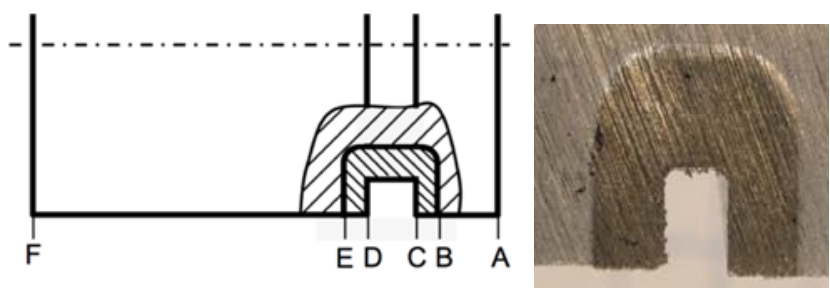

Fig. 3. The phases of turning of the piston's cylindrical surface.
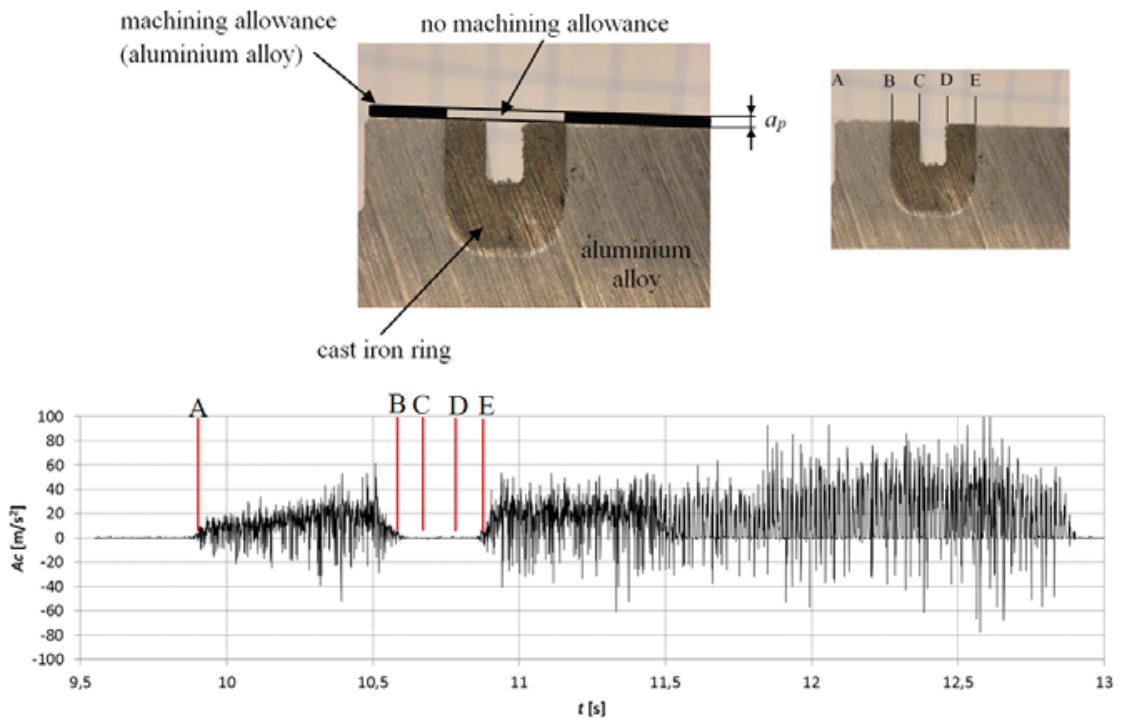

Fig. 4. The collocation of machining allowance and typical vibrations' time course $\left(n=2000 \mathrm{rev} / \mathrm{min}, f=0.6 \mathrm{~mm} / \mathrm{rev}, a_{p}=0.5 \mathrm{~mm}\right)$. 
During the machining of cast iron (BC and DE sections) with the PCD tool, the rapid growth of vibration amplitude is observed, which is further applied to the evaluation of the process condition. The differences in the vibrations level are large enough to carry out the real-time process monitoring.

Figure 6 depicts the control algorithm of the turning correctness, on the basis of the measurement of vibration signals generated during machining. The control process is carried out in the real-time and cyclically for the each manufactured piston.

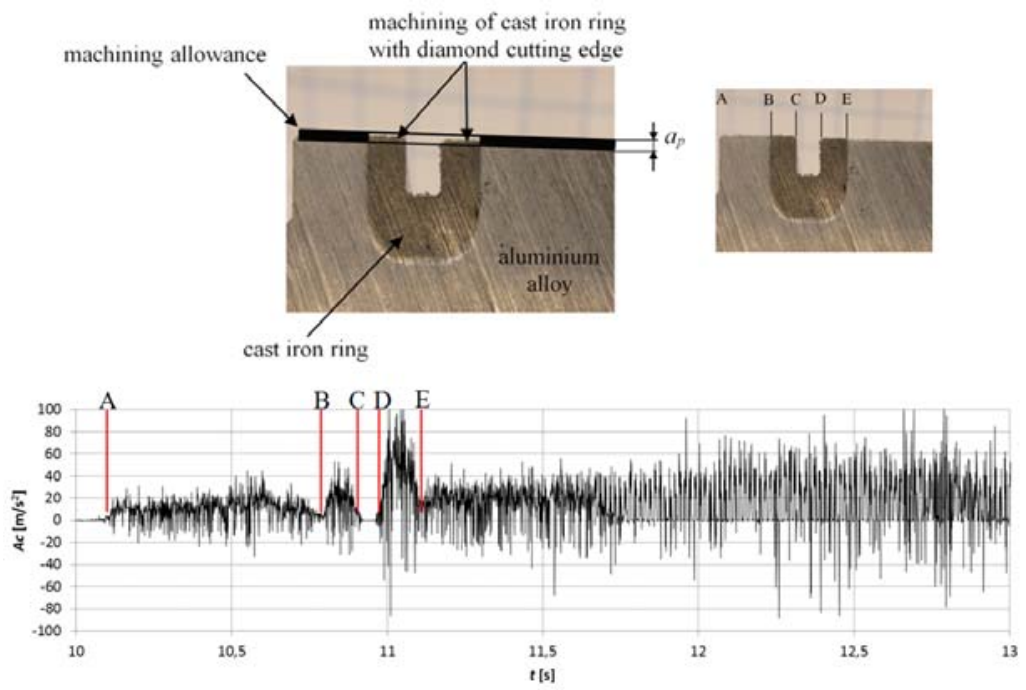

Fig. 5. The incorrect collocation of machining allowance and typical vibrations' time course $\left(n=2000 \mathrm{rev} / \mathrm{min}, f=0.6 \mathrm{~mm} / \mathrm{rev}, a_{p}=0.5 \mathrm{~mm}\right)$.

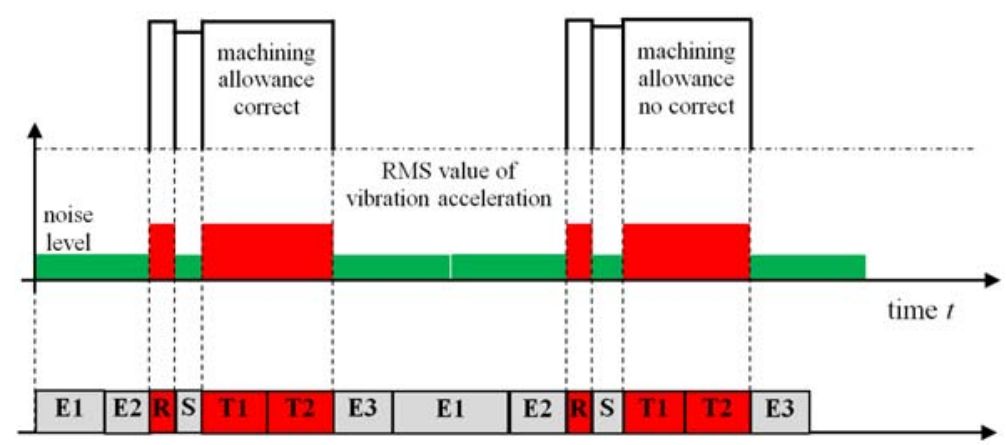

Fig. 6. The control algorithm of the piston's cylindrical surface turning correctness.

The control algorithm is conducted in the following stages:

E1 - The phase of background level detection - the average value of background level is set in the narrow configurable window, moving along the samples, in the time range including the time of the specific phase. The absolute value is determined from the digital signal available in the window. On the basis of this information, the threshold value is being calculated. This value has to be exceeded by the signal's average value in order to recognize this signal as one accompanying the machining process.

E2 - The phase of awaiting the trigger. The signal contained in the specified time window is being preliminarily processed: the removal of constant component and calculation of 
signal's absolute value. The average value of signal is set in the narrow configurable window, moving along the samples, in the time range including the time of the specific phase.

$\mathbf{R}$ - During this stage, the program passes into the awaiting phase of tool's approaching the area of cast iron ring.

$\mathbf{S}$ - Analogically as in E2 phase, the continuous measurement of average vibration values in the set window is conducted. During this stage, the correctness of the piston's production process is evaluated. In case of vibrations with the values averaged in the specified time interval, which significantly exceed the previously set background level, the piston is graded as inappropriately manufactured, otherwise (lower vibration values) the piston is categorized as correctly manufactured.

T1 - The program passes into the awaiting phase during the time needed to the tool's output.

T2 - The signal contained in the specified time window is being preliminarily processed: the removal of constant component and calculation of signal's absolute value. The average value of signal is set in the narrow configurable window, moving along the samples, in the time range including the time of the specific phase.

E3 - During this stage the program is paused for the short time, before the initiation of the next cycle. This pause is needed to the movement of the tool to the starting position, and collecting the next element.

After the execution of the full cycle, the subsequent initiation of the E1 cycle is conducted.

\section{The verification of the proposed system and conclusion}

The proposed monitoring system has been tested for the various conditions: longitudinal turning with fluids, rotational speed $n=1600-2523 \mathrm{rev} / \mathrm{min}$, feed $f=0.6-0.8 \mathrm{~mm}$, depth of cut $a_{p}=0.5-1 \mathrm{~mm}$. Figures 7 and 8 depict two characteristic situations, i.e. when the tool is not cutting in the $\mathrm{BC}, \mathrm{DE}$ areas, and when the cutting occurs in these areas.

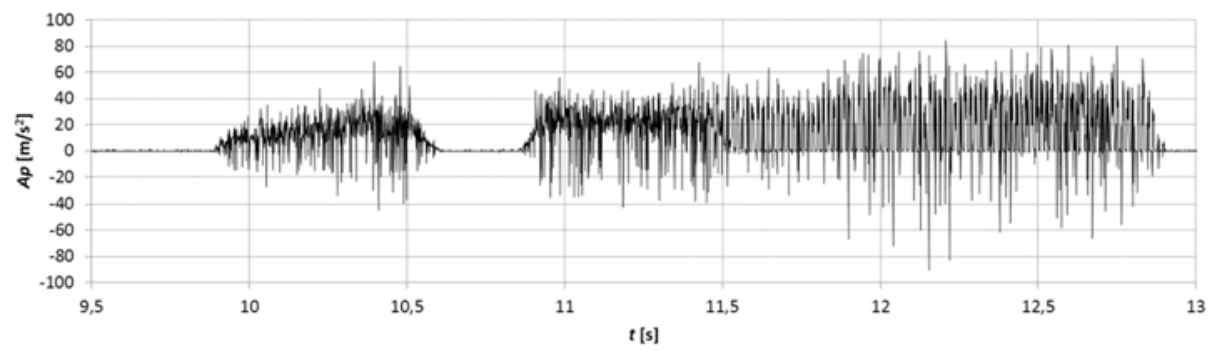

Fig. 7. The exemplary vibration time course, when tool is not cutting in the $\mathrm{BC}$ and $\mathrm{DE}$ areas $\left(n=2000 \mathrm{rev} / \mathrm{min}, f=0.6 \mathrm{~mm} / \mathrm{rev}, a_{p}=0.5 \mathrm{~mm}\right)$.

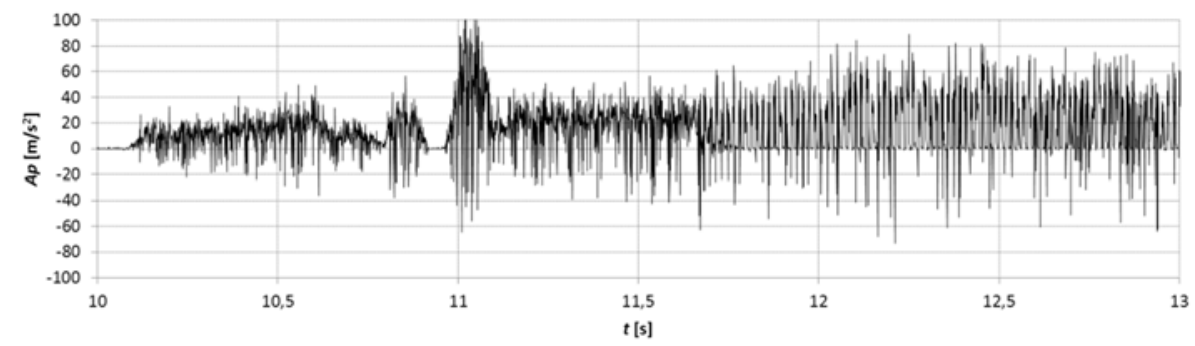

Fig. 8. The exemplary vibration time course, when tool is cutting in the $\mathrm{BC}$ and $\mathrm{DE}$ areas $\left(n=2000 \mathrm{rev} / \mathrm{min}, f=0.6 \mathrm{~mm} / \mathrm{rev}, a_{p}=0.5 \mathrm{~mm}\right)$. 
The presented courses are significantly different. The observation of vibration signals enables the evaluation of machining allowance selection correctness. Turning with the PCD tool in the $\mathrm{BC}$ and $\mathrm{DE}$ areas - besides the negative effect on tool life - very often results in the rupture of the intermetallic bonding between the aluminum alloy and cast iron. In this case the piston is graded as a reject and is consequently intended to the re-casting.

In the each of the analyzed cases, the system correctly identified the condition in which the PCD tool contacted with the cast iron ring. Thus, the expensive rejects have been avoided. Therefore, this system can be successfully applied in the automated production of the IC engine pistons.

\section{References}

1. S. Wojciechowski, R.W. Maruda, P. Nieslony, G.M. Krolczyk, Int J Mech Sci 119, 360-369 (2016)

2. S. Wojciechowski, P. Twardowski, Procedia CIRP 1, 289 - 294 (2012)

3. D. Przestacki, P. Szymanski, S. Wojciechowski, Composites Part A 91 (1), 370-379 (2016)

4. D. Przestacki, M. Jankowiak, Journal of Physics: Conference Series 483 (2014)

5. D. Przestacki, Procedia CIRP 14, 229-233 (2014)

6. S. Wojciechowski, P. Twardowski, M. Pelic, R.W. Maruda, S. Barrans, G. Krolczyk, Precision Eng 46, 158-165 (2016)

7. S. Wojciechowski, P. Twardowski, M. Pelic, Procedia CIRP 14, 113 - 118 (2014)

8. P. Gierlak, A Burghardt, D. Szybicki, M. Szuster, M. Muszyńska, Mechanical Systems and Signal Processing, (On-line)

9. C. Scheffer, H. Kratz, P.S. Heyns, F. Klocke, Int J Mach Tools Manuf 43, 973-985 (2003)

10. C. Scheffer, P.S. Heyns, Mechanical Systems and Signal Processing 18, 1219-1242 (2004) 